should be quoted; unfortunately, this is unknown. We think that it is unwise to base an argument for population screening on the findings in a selected population at risk, particularly when the incidence of abnormality in this selected group is lower than in the population as a whole. This would seem to be yet another example of basing a screening thesis (which is a population based activity) on data generated by secondary and tertiary referral, which is a selected clinical activity.

DAVID M LUESLEY PAUL BYRNE

Department of Obstetrics and Gynaecology, Dudley Road Hospital, Birmingham B18 7QH

\section{Wegener's granulomatosis}

We certainly endorse the message of Dr M G Ridley and others that ear, nose, and throat manifestations of Wegener's granulomatosis are often overlooked ( 30 July, p 352). As such manifestations constitute the presenting symptoms treatment may be delayed while attention is focused on the respiratory tract and "hunting the primary." In 53 patients treated between 1963 and 1988 nasa symptoms were the commonest ear, nose, and throat problems experienced, ranging from mild crusting and epistaxis to septal perforation and collapse of the nasal bridge, though both the ears and the larynx may be affected during the course of the condition.

We do, however, dispute that cyclophosphamide is the only successful cytotoxic agent efficacious in long term remission of these cases. All our patients have been successfully treated with a combination of prednisolone and azathioprine, which can be modulated in response to the erythrocyte sedimentation rate. Azathioprine has proved both effective and virtually free from undesirable complications at daily doses of up to $200 \mathrm{mg} .{ }^{1}$ The therapeutic combination of this cytotoxic agent and steroid is capable of arresting the disease and facilitating repair by fibrosis so it is vital that doctors recognise the condition and institute adequate and expeditious treatment before irreparable pulmonary and renal damage has occurred.

VALERIE J LUND DAVID J HOWARD

\section{Professorial Unit,}

Institute of Laryngology and Otology,

London WCIX 8EE

1 Harrison DFN. Non-healing granulomata of the upper respiratory tract. Br Med f 1974; iv:207-9.

\section{Treating renal calculi}

Mr Nicholas Mays and others (23 July, p 253) compared the results of extracorporeal shock wave lithotripsy and percutaneous nephrolithotomy. Their conclusions supported our policy of recommending percutaneous nephrolithotomy as the primary treatment of most renal and upper ureteric stones. Between January 1985 and April 1988 we treated 141 patients (age range 17-82 years) by percutaneous nephrolithotomy. Stone sizes varied up to $10 \mathrm{~cm}$ in the longest axis, and complete removal was achieved by a single treatment in $89 \%$. In $6 \%$ partial removal was possible, and $5 \%$ were deemed failures because of inadequate visualisation or failed access. Most of the failures were in our first 20 cases. Mortality in the series was zero and major complications occurred in a single patient (severe haemorrhage requiring transfusion).

Minor complications of fever, pain, and infection were observed and are reflected in the time patients stayed in hospital after percutaneous nephrolitho- tomy. The mean length of stay after operation was 5.6 days (range 1.47 days), which compares favourably with the results quoted by the authors. Fifty four per cent of our patients were in hospital for less than five days, and $16 \%$ were discharged within 24 hours. Before June 1987 we routiñely left a nephrostomy tube in position for two or three days. Since then $35 \%$ of our patients have been discharged within 24 hours.

Our patients received a general anaesthetic, and this might be considered a disadvantage when comparing our results with those of the latest lithotripter, but we have not observed any complications resulting from the anaesthetic. We think, therefore, that percutaneous nephrolithotomy is a successful treatment for upper urinary tract stone disease when compared with extracorporeal shock wave lithotripsy. We have referred only a few patients for lithotripsy. These patients had stones in the calices that were deemed inaccessible to percutaneous nephrolithotomy or were patients in whom percutaneous nephrolithotomy had failed We successfully treated one patient who had recently been discharged after lithotripsy to his other kidney, and he preferred percutaneous nephrolithotomy. Few of our patients have had problems after discharge, and all returned rapidly to their usual activities.

A lithotripsy centre has only recently become available in this region, and it is 120 miles from our health district. Previously patients faced a 500 mile return journey, and, given the choice, patients prefer treatment locally after being given full details of the alternatives.

We think that percutaneous nephrolithotomy remains an acceptable and ethical primary treatment for most upper tract stones. A proportion of patients undergoing lithotripsy will require additional interventional treatment, and this often cannot be offered in the lithotripsy centres. Anecdotal evidence suggests that the results of percutaneous nephrolithotomy are much better in centres performing this surgery on a regular sessional basis (probably 2-3 cases each week) We think that on the grounds of efficacy, cost logistics, and patients' convenience there is a strong case for subregional centres of expertise in percutaneous and ureteroscopic stone surgery and that consideration should be given to adequate funding of such centres at the same time as resources are provided for the latest rapidly developing modality of extracorporeal shock wave lithotripsy, the superiority of which is not yet proved.

J C HAMMONDS I P WELLS

Derriford Hospital,

Plymouth PL6 8DH

In their paper on extracorporeal shock wave lithotripsy $\mathrm{Mr}$ Nicholas Mays and others conclude that this method of managing urolithiasis has no obvious advantages over percutaneous nephrolithotomy (23 July, p 253). Although they suggest that their two groups were broadly matched for age, it is worth noting that the youngest in the nephrolithotomy group was aged 12 while the youngest in the lithotripsy group was aged 4. Clearly, therefore, the paediatric age group has not been considered as a separate entity.

It is expected that about 100 children each year will present with urolithiasis in the United Kingdom. ${ }^{\prime}$ Of these, $65 \%$ will be under 5 years with a peak incidence at around 3 years. Few of these children will be suitable for percutaneous nephrolithotomy for technical reasons, and extracorporeal shock wave lithotripsy is therefore a reasonable alternative.

Renal calculous disease in the paediatric age group remains a definite indication for considering lithotripsy. It would be a pity if the conclusions drawn in the article by Mr Mays and others should result in a failure to provide this important service for children.

STEPHEN BROWN

Royal Belfast Hospital for Sick Children,

Belfast BT12 6BE

1 Ghazali S. Childhood urolithiasis in the United Kingdom and Eire. Br J Urol 1975;47:739-43.

Like Mr Nicholas Mays and others (23 July, p 253) we have also had disappointing results with extracorporeal shock wave lithotripsy in a small series of patients. The following is partly taken from our recent presentation at the 1988 meeting of the British Association of Urological Surgeons.

Twenty one consecutive patients from the Swansea area had extracorporeal shock wave lithotripsy in London (two centres, Dornier machines) and were followed up for six months. Sixteen patients with small stones (under $3 \mathrm{~cm}$ in diameter) had extracorporeal shock wave lithotripsy alone: 11 became free of stones, one had "non-surgical" debris, and four had fragments larger than $4 \mathrm{~mm}$ six months later. Two patients with larger stones $(3$ and $4 \mathrm{~cm}$ ) had double $\mathrm{J}$ splints inserted before extracorporeal shock wave lithotripsy; both had residual stones. Two patients with staghorn calculi had preliminary percutaneous nephrolithotomy with satisfactory debulking; at six months both had large (more than $1 \mathrm{~cm}$ ) fragments. The last patient had medullary sponge kidneys and multiple stones, some of which remained after treatment.

In summary, only 13 of the 21 patients $(62 \%)$ with variously sized stones had a satisfactory result from a single treatment with extracorporeal shock wave lithotripsy; eight needed more treatment. These stones had been thought unsuitable for percutaneous nephrolithotomy for various reasons.

By comparison, since starting percutaneous renal operations in June 1986 we have documented 112 percutaneous nephrolithotomies. Altogether 74 out of $106(70 \%)$ achieved complete clearance of stone from the kidney (the six others were debulking procedures).

We look forward to similar reports from other centres referring patients elsewhere for extracorporeal shock wave lithotripsy and suggest that both extracorporeal shock wave lithotripsy and percutaneous nephrolithotomy are necessary for the efficient treatment of renal stones.

ROBERT COX C S POWELL $\mathrm{K}$ C VAUGHTON

M B ROSE MARTIN WHITE

Morriston Hospital Swansea SA6 6NL

\section{Children in Third World slums}

Dr William A M Cutting and Professor Gopa Kothari do well to rub our noses once again in the obscenity of the poverty of Third World slums (18 June, $\mathrm{p}$ 1683). They also do well to send British medical students for a spell to such places. Students will always remember the experience, as they will need to because the problem of world poverty shows little sign of being seriously tackled in their lifetimes, let alone in ours.

I also fervently applaud their remark that "changing clichés will change nothing": nor will anything short of an objective assessment of the facts, including those about breast feeding, supplementation, and manufactured and other breast milk substitutes. Unfortunately, their leading article is not uninfluenced by such clichés. Ninety nine per cent of these children were breast fed They say "there was no difference in nutritional 